\title{
UN PORTAL DE MAGIA CON LA MATEMÁTICA, COMPRENSIÓN DE TEXTOS
}

\section{A PORTAL OF MAGIC WITH MATHEMATICS, UNDERSTANDING OF TEXTS}

Juana Elizabeth Sánchez Machado: Universidad Técnica de Ambato. Ecuador juaniselis@hotmail.com

María Cristina Páez: Universidad Técnica de Ambato. Ecuador mc.paez@uta.edu.ec

Hector Fernando Goméz Alvarado: Universidad Técnica de Ambato. Ecuador hfgomez@uta.edu.ec

Margarita Narváez Rios: Universidad Técnica de Ambato. Ecuador mm.narvaez@uta.edu.ec

Luis Gozalo Maiza Vayas: Universidad Técnica de Ambato. Ecuador lg.maiza@uta.edu.ec

\section{RESUMEN}

Este articulo pretende señalar la influencia que tiene la comprensión lectora en el aprendizaje de la matemática, siendo esta un área donde los estudiantes muestran mayor grado de dificultad, en la primera parte se examinó la importancia de la lectura y su acceso al conocimiento, el mismo que brinda mayor oportunidad para el futuro, formando personas libres. Se señaló que la comprensión lectora es tratada en el área de lengua y literatura, totalmente aislada de las otras materias o áreas del currículo, abandonando la comprensión de textos científicos. El vocabulario matemático es más difícil de entender. El desarrollo de estrategias de comprensión lectora facilita el aprendizaje de los estudiantes, en cualquier área educativa, siendo esta una herramienta para el aprendizaje autónomo.

PALABRAS CLAVE: Comprensión lectora - Aprendizaje matemático - Aprendizaje autónomo - Lengua y literatura - Comprensión de textos científicos -Área educativaHabilidades lingüísticas.

\footnotetext{
1Juana Elizabeth Sánchez Machado: Licenciada en Ciencias de la Educación, mención educación básica por Universidad Técnica de Ambato, y bachiller en la especialidad de físico matemático, tiene el cargo de auxiliar pedagógico en el Instituto Particular Bilingüe "A.B.C."

Correo:juaniselis@hotmail.com
} 
Sánchez Machado, J.; Páez Quinde, M.; Goméz Alvarado, H.; Narváez Rios, M. y Maiza Vayas, L. G. Un portal de magia con la matemática, comprensión de textos.

\section{ABSTRACT}

This article aims to point out the influence of reading comprehension in learning mathematics, this being an area where students show higher degree of difficulty, in the first part the importance of reading and access to knowledge was examined, the same that provides greater opportunity for the future, forming free people. It was noted that reading comprehension is treated in the area of language and literature, totally isolated from other subjects or areas of the curriculum, leaving the understanding of scientific texts. The mathematical vocabulary is harder to understand. The development of reading comprehension strategies facilitates student learning in any educational area, this being a tool for independent learning.

KEY WORDS: Reading comprehension - Mathematical learning - Autonomous learning - Language and literature - Comprehension of scientific texts - Educational area - Language skills.

\section{Como citar el artículo}

Sánchez Machado, J.; Páez Quinde, M.; Goméz Alvarado, H.; Narváez Rios, M. y Maiza Vayas, L. G. (2017). Un portal de magia con la matemática, comprensión de textos. [A portal of magic with mathematics, understanding of texts] Revista de comunicación de la SEECI, 43, 01-14. Doi: http://dx.doi.org/10.15198/seeci.2017.43.1-14 Recuperado de http://www.seeci.net/revista/index.php/seeci/article/view/462

\section{INTRODUCCIÓN}

En esta investigación se evidencia la comprensión lectora y su importancia en el aprendizaje matemático, sabiendo que el lugar característico para el aprendizaje formal de lectura y escritura es la escuela, siendo esta el medio para acceder al conocimiento permanentemente con la cultura escrita y con textos. (Meneses, 2008)

La base del éxito escolar, es el saber leer ya que a través de la lectura se accede al conocimiento, el mismo que brinda mayor oportunidad para el futuro, formando a personas libres. (Sastre, 2015)

El niño para poder desenvolverse en la sociedad debe adquirir habilidades comunicativas, que se desarrollan en la escuela, como las interacciones con sus compañeros, una comunicación eficiente y apropiado para el aprendizaje sin embargo, los niños con habilidades lingüísticas menos desarrolladas pueden tener problemas de conducta y sociales así como las dificultades en lectura. (Villar, Delia; Vieiro, Pilar., 2015)

Desde el punto de vista educativo, la lectura forma parte de los aprendizajes instrumentales por lo tanto, debe ser enseñada con un método determinado, sabiendo que es una actividad cognitiva compleja a través de la cual los signos gráficos se convierten en significados; dicho proceso es relativamente complejo ya que supone la puesta en marcha de procesos perceptivos relacionados con el análisis 
Sánchez Machado, J.; Páez Quinde, M.; Goméz Alvarado, H.; Narváez Rios, M. y Maiza Vayas, L. G. Un portal de magia con la matemática, comprensión de textos.

visual, el acceso al léxico y el acceso al significado. (Luciane, Katya; Silva, Patrícia; Koich, Fabiano, 2016)

La comprensión lectora es una actividad complexa que involucra mecanismos cognitivos, lingüísticos, sociales y emocionales, el éxito escolar se relaciona a una aprensión adecuada del proceso de leer y comprender el texto leído, siendo uno de los requisitos para el aprendizaje. (Guevara, Yolanda; Cárdenas, Karlena; Reyes, Verónica, 2015)

La misma que es tratada en el aula de clase como una disciplina de lengua y literatura totalmente aislada de las otras materias o áreas del currículo, enfocándose únicamente en la comprensión de textos literarios, abandonando la comprensión de textos científicos. (Montes, 2015)

La comprensión de textos literarios son más fáciles de comprender y retener, por lo tanto su contenido asimilable al contexto e imaginación del estudiante, ya que pueden asociarse con experiencias propias del lector mientras que los textos científicos están escritos en prosa expositivo-argumentativa, diseñados para informar y explicar relaciones de fenómenos, lo cual implica un mayor nivel de abstracción y de dominio lingüístico. (Perales, Damian;l Reyes, Rosario ., 2014)

El mismo que es un referente interactivo que se debe entender dentro de un marco comunicativo, como consecuencia, su metaconocimiento incluye la idea de que debe integrar su conocimiento previo acerca del mundo y del tema para lograr construir su propia interpretación coherente del texto, conocimiento implícito y explícito. (Haoming, 2016)

La conciencia morfológica que tiene relación con la semántica (significado de las expresiones lingüísticas), como fonológica (estudia los fonemas o descripciones teóricas de los sonidos vocálicos y consonánticos que forman una lengua) o sintáctica (papel que desempeña una palabra o morfema), y la capacidad de inferencia léxica se entrelazan entre sí para predecir el desarrollo de la comprensión lectora. (Lima, Gustavo; Duarte, Manuel, 2016)

El lector desarrolla esta habilidad de forma superficial especialmente en áreas científicas como las matemáticas, ya que en estas tareas su lectura es superficial es decir mecánica, con énfasis en memorización de palabras, y no lo profundiza. (Guerra, Evelyn; Forero,Carmen, 2015)

Para lograr la comprensión en el proceso lector, es necesario tener en cuenta las características del texto, entre las que se encuentran su direccionalidad y el nivel de comprensión del lector. Otro aspecto destacable es la manera como el texto representa al escritor, contribuyendo a que intercambie significados con el lector. Igualmente, se resalta la sensibilidad del escritor hacia el lector y la forma como utiliza el lenguaje, lo cual facilita la asimilación de significados. (Jiang, Chinese College Students' English Reading Comprehension in Silent, 2015) 
Sánchez Machado, J.; Páez Quinde, M.; Goméz Alvarado, H.; Narváez Rios, M. y Maiza Vayas, L. G. Un portal de magia con la matemática, comprensión de textos.

Lo que permite a que el estudiante pueda construir su vocabulario y establecer un aprendizaje perdurable, lectura-modo silencioso mejora significativamente la comprensión de la lectura, ya que permite que el lector se concentre, mientras que la lectura en voz alta de modo de no revela las mismas conclusiones, ya que prioriza la decodificación de códigos fonológicos, antes que extraer el significado del texto. (Katherine W. Price, Elizabeth B. Meisinger, Max M. Louwerse \& Sidney D'Mello, 2015)

Al leer un texto científico matemático el docente o el estudiante normalmente lo hace con una lectura oral, para que todos puedan escuchar y entender el texto, sin embargo, al tener una lectura oral el nivel de comprensión será más bajo, ya que el lector se concentra en la fluidez de la lectura.

Para que exista una comprensión de textos matemáticos es importante entender el lenguaje matemático y desarrollar competencias, con la introducción de un vocabulario acorde al texto, para así facilitar el proceso de aprendizaje. (Riccomini, Paul J.; Smith, Gregory W.; Hughes, Elizabeth M.; Fries, Karen M. - Reading \& Writing Quarterly., 2015)

La matemática y el aprendizaje de la misma debe estar relacionada con la formación profesional de los estudiantes, tomando en cuenta que existe una gran brecha entre las calificaciones del estudiante y las destrezas que el mismo desarrolla, ya nos podríamos estar encontrando con estudiantes que tienen excelentes calificaciones, pero no aplicaron destrezas propias de la matemática, por lo tanto no tienen un aprendizaje profesional que les permitan desarrollarse en el ámbito laboral. (Huber, Daniel; Jones, Leslie; Helminski, Christine., 2015)

El aprendizaje de matemática, como el desarrollo de destrezas de la misma en particular, se reconoce cada vez más como una herramienta fundamental en la comprensión científica fenómenos, con modelos matemáticos que utilizan disciplinas que van desde las estadísticas a las ecuaciones diferenciales, la geometría es una herramienta eficaz en el modelado biomecánico, y uno que se ha utilizado para desarrollar una serie de lecciones sobre la importancia funcional de las matemáticas en la naturaleza. (Huber, Daniel; Jones, Leslie; Helminski, Christine., 2015)

El progreso y la prosperidad de un país dependen de la calidad de las matemáticas que se enseñan en su sistema escolar mejorar la calidad de vida, habilidades como: la lectura, escritura, aritmética y competencias prácticas, son necesarias para el aprendizaje de las mismas, por lo tanto, podemos decir que una buena comprensión de la matemática es esencial para dar sentido a todos los números y en la solución de los complejos problemas de la vida diaria. (Anjum, Sabahat, 2015)

\section{METODOLOGÍA}

La investigación está enfocada en el modelo pedagógico cognitivo haciendo referencia, a la corriente del paradigma crítico propositivo propio de las ciencias humanas, con el objetivo de ayudar a los y las estudiantes adquirir una educación 
Sánchez Machado, J.; Páez Quinde, M.; Goméz Alvarado, H.; Narváez Rios, M. y Maiza Vayas, L. G. Un portal de magia con la matemática, comprensión de textos.

integral, ampliando su capacidad de razonamiento, reflexión y resolución de problemas matemáticos, preparándolos para afrontar nuevos retos en el futuro.

La investigación es mixta porque se empleó métodos cualitativos y cuantitativos, además se aplicaron técnicas con sus respectivos instrumentos a los estudiantes de séptimo año de Educación General Básica.

Como técnica se utilizó la encuesta y como instrumento un cuestionario estructurado, el objetivo del mismo fue Diagnosticar el nivel de compresión lectora de los niños de séptimo Año de Educación General Básica paralelos "A" y "B" de la Unidad Educativa "Caracas" e identificar el proceso de aprendizaje en el área de matemática mediante el cumplimiento de estándares de calidad.

\section{RESULTADOS}

La lectura en voz alta a temprana edad, permite el acercamiento hacia el conocimiento, estableciendo desde pequeños bases conceptuales, incluso cuando aún no han adquirido el lenguaje oral. (Cortez, Ruth ; Arévalo, Humberto , 2015)

La comprensión lectora es un asunto complejo que implica la contribución teórica de profesionales de diferentes áreas, ya que trabaja en aspectos cognitivos, lingüísticos, textuales y socioculturales, el tener una buena comprensión al inicio de la escolarización, garantiza un rendimiento escolar satisfactorio y el estudiante no tendrá dificultad en el proceso educativo. (Oliveira, Katya Luciane; Lúcio, Patrícia Silva; Miguel, Fabiano Koich. , 2016)

El aprendizaje de la lecto-escritura de una lengua no se da en forma natural, ya que necesita de la mediación de ambientes educativos formales; además, está configurada por estructuras verbales complejas, que cobran significados específicos en contextos determinados y es propia de la cultura de las personas.

Los lectores con dificultades de comprensión lectora tienen fortalezas, que utilizan para compensar sus dificultades, algunos estudiantes, tienen buenos recursos en percepción del habla, reconocimiento inmediato de palabras, velocidad de nombrado y memoria de trabajo lo cual se puede asociar a la capacidad de aprender y emplear estrategias para recordar información. (Martha Lucia Rincón-Bustos, Ángela AguirreBravo, Saida Melisa Carmona, Paula Contreras-Ruiz, Laura Figueredo-Higuera, Constanza Guevara-Urrego, Sandra Liliana Sosa-Sabogal, Ana Jasmin Urán-Loaiza, 2015)

La motivación juega un papel importante en la comprensión de textos, así como en los procesos cognitivos, ya que permite que el lector tenga seguridad en sí mismo, aumentando su interés y deseo en el tema, cuando existen índices de ansiedad disminuye la comprensión lectora. (Aysel, Memiş; Metin, Bozkurt, 2013)

Los padres y maestros juegan un papel fundamental, ya que la actitud de apoyo que tengan con los estudiantes, directa o indirectamente motivan al estudiante a crear 
Sánchez Machado, J.; Páez Quinde, M.; Goméz Alvarado, H.; Narváez Rios, M. y Maiza Vayas, L. G. Un portal de magia con la matemática, comprensión de textos.

un hábito lector, siendo los mismos, predicadores con su ejemplo. (Lim, Hyo Jin; Bong, Mimi; Woo, Yeon-Kyung, 2015)

Los matemáticos y profesores de matemáticas en sí, son buenos lectores de textos, de cualquier tema que este sea, mientras que los maestros especializados han otra área, se han centrado su aprendizaje y enseñanza sobre la lectura de ficción y prosa, por lo tanto la matemática es particularmente difícil de leer el contenido, la presentación de demandas en el pensamiento y las habilidades de comprensión de nivel más alto, muchos estudiantes no pueden comprender fácilmente textos de matemáticas, incluso cuando son capaces de decodificar los materiales de impresión, estos libros hacen un uso intensivo de símbolos precisos y vocabulario desconocido, utilizan estructuras de la oraciones más largas y complejas; contienen más palabras, símbolos y conceptos por párrafo que otros textos; y tienen poca redundancia para ayudar con la interpretación. (Adams,Anne; Pegg,Jerine ; Case, Melissa., 2015)

Se debe tener en cuenta que el desarrollo de las habilidades metacognitivas no sólo va ligado a la madurez biológica, sino que está directamente influido por las diversas experiencias de aprendizaje del sujeto, ya que son éstas las que posibilitan, en mayor o menor grado, el nivel de conocimiento que posee el sujeto sobre el proceso de lectura. (Fernández,Eduardo; Núñes, Rocío; Fernández, María, 2015)

La lectura compartida permite al lector compartir su criterio con los demás, ampliando así su conocimiento, mejorando su rendimiento académico, aumentando y haciendo uso del vocabulario nuevo y fomentando la práctica de la lectura por placer. (Goikoetxea, Edurne ; Martínez, Naroa , 2015)

El uso de las tecnologías beneficia al lector y permite que tenga acceso a libros, facilitando así su lectura autónoma y por ende el aprendizaje basado en el constructivismo ya que el estudiante crea su propio conocimiento. (Ortega, 2015)

Es importante que en la enseñanza de matemáticas no se presione a los estudiantes, ya que el ambiente en el cual ellos aprenden es fundamental para el acercamiento al aprendizaje de las matemáticas. (Gonzales, 2016)

En la práctica del área de matemática se debe enfatizar los objetivos y contenidos, los mismos que deben ser claramente establecidos para que orienten la práctica educativa de un país, a partir de recursos múltiples. (Ruiz, 2015)

Los primeros años de vida son fundamentales en el desarrollo de habilidades matemáticas, pero las oportunidades de aprendizaje a esa edad son limitadas por el contexto socio económico de los habitantes que uno tiene, lo que impide el desarrollo de un aprendizaje significativo. (Wang, Aubrey H.; Firmender, Janine M.; Power, Joshua R.; Byrnes, James P., 2016)

\section{Discusión}

Para desarrollar un aprendizaje autónomo eficiente y eficaz en el área de matemáticas es imprescindible que el estudiante desarrolle destrezas lingüísticas, 
Sánchez Machado, J.; Páez Quinde, M.; Goméz Alvarado, H.; Narváez Rios, M. y Maiza Vayas, L. G. Un portal de magia con la matemática, comprensión de textos.

como es la comprensión lectora. La misma que empieza desde una edad temprana esto favorecerá a que adquiera un hábito lector, además permite que se adquiera nuevo vocabulario, ampliando así su conocimiento.

La comprensión lectora permite que el estudiante comprenda lo que lee, que trabaje de manera autónoma, desarrollando juicios de valor sobre el texto, brindándole oportunidades en su vida personal y profesional.

La matemática nos ayuda a desarrollar nuestro pensamiento crítico, racional, propositivo, para resolver problemas de la vida diaria, permitiéndonos desenvolvernos en la sociedad.

Es necesario que se sigan realizando investigaciónes sobre el tema, ya que permitirá que los docentes y en si en la escuela se fusione la comprensión lectora con las demás áreas en el currículo, permitiendo al docente realizar su labor más fácil sin abandonar esta destreza que es imprescindible para que los estudiantes puedan aprender, eliminando creencias negativas en el área de matemática. Es necesario que se involucre a la familia en la escuela ya que son el pilar fundamental de motivación del estudiante.

\section{Conclusiones}

La comprensión lectora involucra mecanismos, no solo de conocimientos previos sino también, de comunicación, sociales y emocionales, que pueden afectar o beneficiar al desarrollo de habilidades lingüísticas y por ende al aprendizaje de las mismas.

El leer cuentos desde el embarazo ayuda a la estimulación de la actividad cerebral, mostrándoles otras experiencias y acercándolos al conocimiento, siendo esta la base para la adquisición de destrezas futuras en el proceso escolar, además fomenta hábitos lectores en todas las etapas de su vida.

La motivación despierta el interés de los estudiantes hacia la lectura, ya este factor es el que impulsa al ser humano a que pueda alcanzar sus objetivos, si no existe esa predisposición para lograr una actividad difícilmente se logra cumplir con las metas trazadas.

El vocabulario matemática es más difícil de entender que el vocabulario cotidiano, por ende debe existir un vocabulario enriquecido en esta área para poderlo entender, mayoritariamente los estudiantes memorizan conceptos matemáticos para rendir en las evoluciones.

El desarrollo de estrategias de comprensión lectora facilita el aprendizaje de los estudiantes, en cualquier área educativa, siendo esta una herramienta para el aprendizaje autónomo. 
Sánchez Machado, J.; Páez Quinde, M.; Goméz Alvarado, H.; Narváez Rios, M. y Maiza Vayas, L. G. Un portal de magia con la matemática, comprensión de textos.

\section{REFERENCIAS}

Adams,A.; Pegg, J. ; Case, M. (2015). Anticipation Guides: Reading for Mathematics Understanding. Mathematics teacher, 108(7)

Anjum, Sabahat. (2015). Gender Difference in Mathematics Achievement and Its Relation with Reading Comprehension of Children at Upper Primary Stage. Journal of Education and Practice, 6, 16, 71-76

Backhoff, E., Sánchez, A., Peón, M., \& Andrade, E. (2010).). Comprensión lectora y habilidades matemáticas de estudiantes de educación básica en México: 20002005. REDIE: Revista Electrónica de Investigación Educativa, 12 (1), 18 p.

Barba, L. (2002). Pedagogia y relacion educativa. México.

Becerra, J. (2012). Destrezas lectoras. En J. Becerra, Lenguaje y comunicacion. Quito: Holos editorial.

Bofarull, T. (2001). Comprension lectora. España: Editorial Laboratorio Educativo.

Bolivar, A. (2011). Competencias Basicas: razones. España: editorial Sintesis.

Bosco, D. (2000). Tipos de lectura. en D. Bosco, Lenguaje y Comunicacion. Quito: Editorial Don Bosco.

Bustos, E. (2010). Dificultades en la comprension lectora. Innovacion y experiencias educativas, 37, 1-10

Cañuelo, C. (2011). La dislexia.net. Recuperado de http://www.ladislexia.net/dificultades-de-comprension-lectora/

Caravajal, L. (2013,). Lizardo Caravajal.com. Recuperado de http://www.lizardocarvajal.com/que-es-la-lectura/

Carrasco, J. B. (2004). Una didactica para Hoy. Madrid: Ediciones Rialp S.A.

Catalá G; Catala M.; Molina E.; Monclus R. (2001). Componentes de la comprension lectora. En C. M. Catalá Gloria, Evaluacion de la comprension lectora (p. 221). Barcelona: Imprimix.

Centro Virtual Cervantes. (2016). Centro Virtual Cervantes . Recuperado de http://cvc.cervantes.es/ensenanza/biblioteca ele/diccio ele/diccionario/comprens ionlectora.htm

Chicaiza, D. (2012). Estrategias de lectura comprensiva y su incidencia en el aprendizaje significativo de los estudiantes de tercer año de Educacion Basica de la escuela Humberto Ochoa. Ecuador: Universidad Técnica e Ambato. 
Sánchez Machado, J.; Páez Quinde, M.; Goméz Alvarado, H.; Narváez Rios, M. y Maiza Vayas, L. G. Un portal de magia con la matemática, comprensión de textos.

Cortez, R. ; Arévalo, J. H . (2015). Dificultades lingüísticas en la lectura en voz alta en niños del grado 1-4 de la sede Santo Tomás de la institución educativa municipal Santa Teresita de Catambuco de Pasto. Tendencias, XVI, 1, 65-75.

Diaz Ortíz, A. (2010). Comprension lectora de los aprendizajes matematicos. Innovacion y experiencias educativas, 27, 1-14.

Duque, S. (2014). Resultados de las pruebas "Ser Estudiante" serán un insumo para la política pública. El ciudadano.

El Comercio. (2012, Noviembre, 28). El habito de la lectura es abandonado por los ecuatorianos.

El Comercio. (2012, Diciembre, 14). Ambato es una de las ciudades con más alto indice de lectura .

El Comercio. (2012, Diciembre, 14). Ambato es una de las ciudades con el más alto índice de lectura.

El Comercio. (2014, julio, 03). 'Bomberos' en la evaluación Ser Estudiante que se aplicó a los alumnos del Ecuador.

El Comercio. (2014, julio 03). La evaluación Ser Estudiante que se aplicó a los alumnos del Ecuador.

El Diario Manabi. (2007, septiembre, 18). Lectura comprensiva o leer entrelíneas. El diario.

Facultad de ciencias de la educacion. (2015). I.exam-10.com. Recuperado de http://l.exam-10.com/doc/10683/index.html

Fernández, E.; Núñes, R.; Fernández, M. (2015). Aportaciones de un análisis de necesidades sobre la situación. Revista de Investigación en Educación, 13(2), 288-302

Flores Macías, R. C.; Jiménez, J. E. y García E. (2015). Basic Cognitive Processes Associated with Secondary Students' Difficulties in Reading Comprehension. Revista Mexicana de Investigación Educativa, 20(65), 581-605

Fuentes, L. (2009). Diagnostico de la comprension lectora en Educacion Básica en Villarica. Perfiles educativos, 31(125), 23-37

Garcia, J. A. (2015). El Lenguaje Ordinario: La Clave para el Aprendizaje de las Matemáticas Basado en Problemas. Actualidades Investigativas en Educación, 15, $1,495-519$ 
Sánchez Machado, J.; Páez Quinde, M.; Goméz Alvarado, H.; Narváez Rios, M. y Maiza Vayas, L. G. Un portal de magia con la matemática, comprensión de textos.

Goikoetxea, E, ; Martínez, N. . (2015). The benefits of shared book reading: a brief review. Educación XX1: Revista de la Facultad de Educación, 18, 1, 303-324

Gonzales, L. (2016). Elaboración y evaluación de "tareas matemático-literarias" para mejorar la comprensión en 30 de la ESO. Tesis doctoral. Universidad de Extremadura

Guerra, Evelyn; Forero,Carmen. (2015). Estrategias para la comprensión de textos academicos. Zona Próxima, 22, 33 - 55.

Guevara, Y.; Cárdenas, K.; Reyes, V. (2015). Niveles de comprensión lectora en alumnos de secundaria. Una comparación por tópico. Actualidades en Psicología, $29,118,13-24$

Haoming, Z. (2016). Concurrent and Longitudinal Effects of Morphological Awareness on Reading Comprehension Among Chinese-Speaking Children. Reading Psychology, 37, 6, 867-884.

Huber, D.; Jones, L.; Helminski, Christine. (2015). The Importance of Mathematical Models to Scientific Discovery: A Case Study on the Feeding Mechanism of the Goliath Grouper "Epinephelus itajara". Australian Mathematics Teacher, 71, 3, 24-29.

INEC. (2012). Hábitos. Quito.

Jiang, Y. (2015). Chinese College Students' English Reading Comprehension in Silent. English Language Teaching, 8, 4, 24-30.

Katherine W. Price, Elizabeth B. Meisinger, Max M. Louwerse \& Sidney D'Mello. (2015). The Contributions of Oral and Silent Reading Fluency to Reading Comprehension. Reading Psychology, 37, 2, 167-201.

Lim, Hyo Jin; Bong, Mimi; Woo, Yeon-Kyung. (2015). Reading Attitude as a Mediator between Contextual Factors and Reading Behavior. Teachers College Record, $117,1$.

Lima, Gustavo; Duarte, Manuel. (2016). Concepções de estudantes. Ensaio: Avaliação e Políticas Públicas em Educação, 24, 91, 380-394

LOEI. (2008). LOEI.Quito.

Lozano, C. S. (2009). Comprension textual. Colombia: Kimpres Ltda.

Luciane, Katya; Silva, Patrícia; Koich, Fabiano. (2016). Considerations about Understanding ability in reading and Ways of its Assessment. Psicologia Escolar $e$ educacional, 20 (1) 10. 
Sánchez Machado, J.; Páez Quinde, M.; Goméz Alvarado, H.; Narváez Rios, M. y Maiza Vayas, L. G. Un portal de magia con la matemática, comprensión de textos.

Memis, A.; Bozkurt, M. (2013). The relationship of reading comprehension success. Educational Research and Reviews, 8, 15, 1242-1246.

Mendonça, D. ; Sella, A.; Motta, S. ; Motta, H . (2016). Assessing story production and retelling repertoires in children. Estudos de psicologia. Natal, 21 (1)

Meneses, A. (2008). Leer y escribir en una escuela chilena. Signos, 22, 257-278.

Mialaret, G. (1979). El derecho del niño a la educacion. Paris: Imprenta de Presses Universitaires de France, Vendome.

Ministerio de Educación. (2011). In E. d. Aprendizaje, Estandares de Calidad Educativa. Quito: Ministerio de Educacion.

Ministerio de Educación.(2011). Estandares de Aprendizaje. In M. d. Educación, Estandares de Calidad Educativa. Quito: Ministerio de Educacion.

Ministerio de Educacion.(2010). Objetivos matematicos. In M. d. Educacion, Actualizacion y fortalecimiento curricular. Quito.

Ministerio de Educación. (2010). Actualizacion y fortaleciemiento curricular. Quito

Ministerio de Educación. (2011). LOEI. Quito.

Ministerio de Educación. (2016). Curriculo. Bogota, Colombia.

Miranda, H. (2013, Noviembre 16). ¿Como evaluar los nieveles de comprension lectora?

Montes, P. (2015). Niveles de comprensión lectora en alumnos de secundaria. Una comparación por tópico. Actualidades en Psicología, 29(118), 13-23. Doi:http://dx.doi.org/10.15517/ap.v29i118.14619

Murga, P. (2015). Diccionarios Rioduero. Madrid: Ediciones Rioduero.

Oliveira, Katya Luciane; Lúcio, Patrícia Silva; Miguel, Fabiano Koich. . (2016). Considerações Sobre a Habilidade de Compreensão em Leitura e Formas de sua Avaliação. Psicologia Escolar e Educacional, 20(1), 69-77. Doi:http://dx.doi.org/10.1590/2175-353920150201930

Ortega, F. (2015). La importancia de la lectura y de las nuevas tecnologías en el aprendizaje del español para inmigrantes. Investigaciones Sobre Lectura, 3, 123133

Ortiz, A. (2013). Modelos pedagogicos y teorias del aprendizaje. Bogota: Editorial Buena Semilla. 
Sánchez Machado, J.; Páez Quinde, M.; Goméz Alvarado, H.; Narváez Rios, M. y Maiza Vayas, L. G. Un portal de magia con la matemática, comprensión de textos.

Pasquel, V. (2010). La comprensión lectora en el proceso de enseñanza aprendizaje de los alumnos del terce año de educacion basica de la escuela "Abdon Calderon". Ambato, Ecuador: Universidad Técnica de Ambato.

Perales, D.; Reyes, R. (2014). The impact of a linguistic intervention on rhetorical. Signos, 23.

Pérez, E. J. (2014). Comprensión lectora VS Competencia. Investigaciones sobre lectura, $1,65-74$

Pulgar, J. (2005). Evaluación del aprendizaje en educación no formal. Madrid: Narcea.

Quesada, J. (2016). Niveles de Comprension Lectora. ecured.

Ramos, L. (2010). Psicologos.com. Recuperado de: http://www.psicopedagogia.com/articulos/?articulo=316

Riccomini, P. J.; Smith, G. W.; Hughes, E. M.; Fries, K M. The Language of Mathematics: The Importance of Teaching and Learning Mathematical Vocabulary. Reading \& Writing Quarterly, 31(3), 235-252. doi:10.1080/10573569.2015.103099

Rincón Bustos M. L.; Aguirre Bravo, A.; Carmona, S. M.; Contreras Ruiz P.; Figueredo Higera L.; Guevara Urrego, C.; Sosa Sabogal S. L.; Urán Loaiza, A. J. (2015) ¿Cómo la comprensión de lectura en estudiantes sordos se ve facilitada por el uso de tecnologías de la comunicación e información? Revista de la Facultad de Medicina. 63-91. doi: http://dx.doi.org/10.15446/revfacmed.v63n3sup.50570.

Roca, D. J. (2015). Hiperlexia: Mitos y Realidades. Encolombia.

Ruiz, A. (2015). Los "estándares" en la educación matemática de los Estados Unidos. Uniciencia, 20, 2, 379-391.

Santillana. (2010). Textos. Quito: Santillana.

Santillana, G. (2011). Aprendizaje matematico. In G. Santillana, ¿Como evaluar e/ area de matematicas? Quito: Santillana.

Sastre, M. S. (2015). La lectura y la competencia lectora en el siglo XXI. Investigaciones Sobre Lectura, no 5, 81-83.

TIC, E. (2006). Dificultades en el aprendizaje. In J. F. Cerván, Dificultades en el aprendizaje: unificación de criterios diagnósticos. Sevilla: Junta de Andalucía

Tomas, U. d. (2014). Curriculo. Universidad de Santo Tomas. 
Sánchez Machado, J.; Páez Quinde, M.; Goméz Alvarado, H.; Narváez Rios, M. y Maiza Vayas, L. G. Un portal de magia con la matemática, comprensión de textos.

Tomas, U. S. (2016). Tipos de modelos pedagogicos. Santo Tomas, 10.

Tusquets, J. (1983). Teroria de la educacion. Madrid: Maggisterio Españos S.A.

Villar, D. yVieiro, P. (2015). Métodos de lectura y acceso léxico on-line en lectores principiantes. Ciencias Psicológicas, 9(2), 309 - 319.

Wang, A. H.; Firmender, J. M.; Power, J. R.; Byrnes, J. P. (2016). Understanding the Program Effectiveness of Early Mathematics Interventions for Prekindergarten and Kindergarten Environments: A Meta-Analytic Review. EarlyEducation and Development, 27, 5, 692-713

Ward, W. (2010). Consejos para Ayudar a Niños con Dificultades. Super Duper $\AA$ Handy Handouts! ${ }^{\circledR}, 139$.

\section{AUTORES:}

\section{Juana Elizabeth Sánchez Machado}

Licenciada en Ciencias de la Educación mención Educación Básica por Universidad Técnica de Ambato, y bachiller en la especialidad de físico matemático, tiene el cargo de auxiliar pedagógico en el Instituto Particular Bilingüe "A.B.C.".

\section{María Cristina Páez}

Ingeniera en Sistemas y Computación, Magister en Tecnologías para la Gestión y Práctica Docentes, Docente Investigadora de la Facultad de Ciencias Humanas y de la Educación, Directora Académica Administrativa de la Maestría en Informática Educativa, Design of a semanticframework to modeling human behavior in survillancecontext. Revista: Scopus

https://www.researchgate.net/profile/Maria Paez13

\section{Hector Fernando Goméz Alvarado}

PhD. en Informática por la Universidad Nacional de Educación a Distancia, Experto en sistemas de Información Geográfica, Universidad Internacional de Andalucía. PostDoc en la ETS-Canadá. Ingeniero en Informático en la Universidad Técnica Particular de Loja-Ecuador. Docente investigador y Director de Postgrados a tiempo completo en la Universidad Técnica de Ambato.

http://orcid.org/0000-0002-7310-4260

\section{Margarita Narváez Rios}

Ingeniera en Sistemas, Asistente de Tics en la Universidad Técnica de Ambato

\section{Luis Gonzalo Maiza Vayas}

Analista de sistemas académicos, Licenciado en Ciencias de la Educación mención 
Sánchez Machado, J.; Páez Quinde, M.; Goméz Alvarado, H.; Narváez Rios, M. y Maiza Vayas, L. G. Un portal de magia con la matemática, comprensión de textos.

Educación Básica, Analista de sistemas académicos, Subdirector del Centro Escolar Ecuador, Docente secundario y Docente de educación básica. 